\title{
A new method of identification of constitutive equations according to the results of technological experiments
}

\author{
O.P. Tulupova ${ }^{1, \dagger}$, V.R. Ganieva ${ }^{1}$, A.A. Kruglov ${ }^{1,2}$, F.U. Enikeev ${ }^{1}$ \\ †box_mail_2011@mail.ru \\ ${ }^{1}$ Ufa State Petroleum Technological University, 1 Kosmonavtov St., 450062, Ufa, Russia \\ ${ }^{2}$ Institute for Metals Superplasticity Problems, 39 Khalturina St., 450001, Ufa, Russia
}

\begin{abstract}
A method is proposed which allows one to search for a solution to inverse problems of identifying constitutive relations from the results of technological experiments. The method uses simplified mathematical models of technological plastic metal forming processes based on the membrane theory of shells, which allows for calculations of material constants $K$ and $m$ entering Backofen's constitutive relation for superplasticity from the results of experiments carried out directly on a technological equipment. The method differs from others by the fact that for its implementation results of only two test formings of hemispherical domes at a constant gas pressure are sufficient. The set of experimental data include gas pressure, forming time and thickness in the pole of the dome. The method consists of three steps: calculation of initial material constants $K$ and $m$; fitting of coefficient $m^{*}$ until the coincidence of computed value of the thicnkness at the dome pole with experimentally determined value; correction of the value of constant $K^{*}$. Finite-elements simulations of superplastic forming (SPF) are done by means of ANSYS software. Computational model of material is considered in the framework of creep theory. Time dependencies of relative dome height and relative thickness of semispheres at the dome pole using approximate formulas and simulations using ANSYS are plotted. The method is verified on an example of superplastic forming of semispheres with $35 \mathrm{~mm}$ radius from sheet blanks of titanium alloy VT6 (analogue of Ti-6Al-4V) with thickness of $1 \mathrm{~mm}$ to a cylindric matrix with diameter $70 \mathrm{~mm}$ and height $35 \mathrm{~mm}$ with entry radius of $1 \mathrm{~mm}$ at two constant values of gas pressure 0.5 and $0.7 \mathrm{MPa}$. The forming temperature was equal to $900^{\circ} \mathrm{C}$. As a result of validation, agreement of experimental and computational data acceptable for engineering calculations has been obtained.
\end{abstract}

Keywords: superplastic forming, modeling, material constants, titanium alloy, ANSYS.

\section{Новая методика идентификации определяющих соотношений по результатам технологических экспериментов}

\author{
Тулупова О.П. ${ }^{1, \dagger}$, Ганиева В.Р. ${ }^{1}$, Круглов А.А. ${ }^{1,2}$, Еникеев Ф.У. ${ }^{1}$ \\ 'Уфимский государственный нефтяной технический университет, ул. Космонавтов, 1, 450062, Уфа, Россия \\ ${ }^{2}$ Институт проблем сверхпластичности металлов РАН, ул. Ст. Халтурина, 39, 450001, Уфа, Россия
}

Предложена методика, позволяющая искать решение обратных задач идентификации определяющих соотношений по результатам технологических экспериментов. В методике использованы разработанные упрощенные математические модели технологических процессов обработки давлением, построенные на основе безмоментной теории оболочек, позволяющие вычислять постоянные материала $K$ и $m$, входящие в определяющее соотношение сверхпластичности Бекофена, по результатам экспериментов, которые проводятся непосредственно на технологическом оборудовании. Методика отличается от известных, тем, что для ее реализации достаточно результатов только двух тестовых формовок полусферических куполов при постоянном значении давления газа. Набор экспериментальных данных включает: давление газа, время формовки и толщину в полюсе купола. Методика состоит из трех этапов: расчет начальных значений постоянных материала $K$ и $m$; подбор величины показателя $m^{*}$ до совпадения расчетного значения толщины в полюсе купола с экспериментально полученным значением; корректировка величины постоянной $K^{*}$. Конечно-элементное моделирование процесса СПФ выполнено в среде программного комплекса ANSYS. Численная модель материала рассматривается в рамках теории ползучести. Построены временные зависимости относительной высоты купола и относительной толщины в полюсе купола полусфер по приближенным формулам и в среде ANSYS. Методика проверена на примере сверхпластической формовки при двух постоянных значениях давления газа 0,5 и 0,7 МПа полусфер радиусом 35 мм из листовых заготовок титанового сплава ВТ6 (аналог Ti-6Al-4V) толщиной 1 мм в цилиндрическую матрицу диаметром 70 мм и высотой 35 мм с входным радиусом 1 мм. Температура формовки $900^{\circ} \mathrm{C}$. В результате апробации методики было получено приемлемое для инженерных расчетов согласие экспериментальных и расчетных данных.

Ключевые слова: сверхпластическая формовка, моделирование, постоянные материала, титановый сплав, ANSYS. 


\section{1. Введение}

Сверхпластическая формовка (СПФ) является одним из эффективных процессов обработки давлением трудно-деформируемых листовых материалов. Изделия, полученные СПФ используются в различных областях промышленности, в том числе в нефтегазовой, например: топливные баки, поплавки контакторы уровней, радиаторы и т.д.

В работе представлена новая методика, позволяющая определять постоянные материала, входящие в стандартное степенное соотношение сверхпластичности (СП), предложенное Бекофеном [1]:

$$
\sigma=K \xi^{m}
$$

где $\sigma$ - напряжение течения; $\xi$ - скорость деформации; $K$ - константа материала, зависящая от температуры, среднего размера зерен и других структурных параметров; $m-$ коэффициент скоростной чувствительности материала.

От определения значений постоянных материала $K$ и $\mathrm{m}$ зависит адекватность математических моделей технологических процессов СП. Традиционный метод их вычисления включает серию испытаний на одноосное растяжение стандартных образцов при разных скоростях деформации [2]. В работе [3] предложена методика определения постоянных материала СП по результатам натурных экспериментов, состоящих из трех тестовых формовок полусфер в цилиндрическую матрицу. Оценка точности моделей процесса СПФ, созданных с использованием постоянных материала, определенным по разным методикам дана в работе [4]. Показано, что при определении постоянных $K$ и $m$ из натурных экспериментов достигается приемлемое для инженерных расчетов согласие результатов моделирования с экспериментом.

Вопросы определения постоянных материала СП К и $\mathrm{m}$ рассмотрены также в работах [5-7].

Предлагаемая методика определения постоянных материала $K$ и $m$ из соотношения (1) позволяет снизить необходимое количество натурных экспериментов до двух тестовых формовок полусфер в цилиндрическую матрицу. Кроме того, в отличие от методики [3], предлагаемая методика позволяет прогнозировать не только высоту купола полусферы, но и толщину в полюсе.

\section{2. Математическое моделирование}

На рис. 1 представлена расчетная схема математической модели СПФ полусферы. Применены следующие обозначения: $R-$ текущий радиус купола; $R_{0}-$ радиус цилиндрической матрицы; $r_{0}-$ входной радиус матрицы; $\alpha-$ угол между осью симметрии и текущим радиусом купола, проведенным к центру кривизны входного радиуса матрицы.

Для апробации методики использовали экспериментальные данные СПФ при постоянном давлении полусфер высотой $H=35$ мм из листов титанового сплава BT6 толщиной $s_{0}=1$ мм в матрицу радиусом $R_{0}=35$ мм, имеющей входной радиус $r_{0}=1$ мм [3] (см. табл. 1). Тем- пература формовки $900 \pm 2^{\circ} \mathrm{C}$.

Предоставленная методика состоит из трех этапов.

\section{1. Этап 1 - Расчет начальных значений постоянных $K_{0} u m_{0}$}

Расчет начальных значений постоянных материала $K$ и $m$ проводим согласно методики [8]. Значение $m$ находим численными методами путем минимизации выражения:

$$
\Phi(m)=\left[\frac{t_{1}}{t_{2}} \cdot\left(\frac{s_{02} p_{1}}{s_{01} p_{2}}\right)^{1 / m}-\frac{I_{m}^{\prime}\left(\alpha_{1}\right)}{I_{m}^{\prime}\left(\alpha_{2}\right)}\right]^{2} \rightarrow \min ,
$$

где $I_{m}^{\prime}-$ функция двух переменных $m$ и $\alpha$, представляющая собой следующий определенный интеграл:

$$
I_{m}^{\prime}(\alpha)=\int_{0}^{\alpha}\left(\frac{1}{x}-\operatorname{ctg} x\right) \cdot\left[\frac{\sin ^{3} x}{x^{2}} \cdot \frac{1}{1-r_{0}^{\prime} \sin \alpha}\right]^{1 / m} d x,
$$

где $r_{0}^{\prime}=r_{0} /\left(R_{0}+r_{0}\right)-$ безразмерный входной радиус матрицы, $\alpha-$ угол между осью симметрии и текущим радиусом купола.

Интеграл (3) вычисляем также численными методами. Значения $K_{1}$ и $K_{2}$ определяем по формулам:

$$
\begin{aligned}
& K_{1}=\frac{p_{1}\left(R_{0}+r_{0}\right)}{2 s_{01}} \cdot\left[\frac{t_{1}}{2 I_{m}^{\prime}\left(\alpha_{1}\right)}\right]^{m}, \\
& K_{2}=\frac{p_{2}\left(R_{0}+r_{0}\right)}{2 s_{02}} \cdot\left[\frac{t_{2}}{2 I_{m}^{\prime}\left(\alpha_{2}\right)}\right]^{m} .
\end{aligned}
$$

$K_{0}$ определяем как среднеарифметическое:

$$
K_{0}=\left(K_{1}+K_{2}\right) / 2 .
$$

В результате расчетов получаем: $m_{0}=0,43$ и $K_{0}=413,94 \mathrm{M \Pi a} \cdot \mathrm{c}^{m}$.

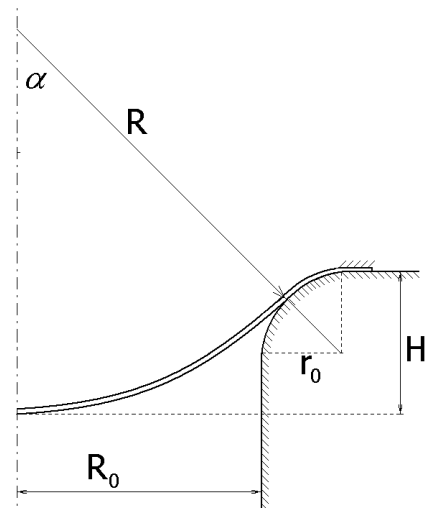

Рис. 1. Расчетная схема.

Fig. 1. Diagram.

Табл. 1. Экспериментальные данные СПФ полусфер.

Table 1. Experimental data of SPF hemispheres.

\begin{tabular}{|c|c|c|}
\hline $\begin{array}{c}\text { Давление, } p, \text { MПа } \\
\text { Pressure, } p, \text { MPa }\end{array}$ & $\begin{array}{c}\text { Время, } t, \text { с } \\
\text { Time, } t, \text { sec }\end{array}$ & $\begin{array}{l}\text { Toлщина в полюсе купола, } s, \text { мм } \\
\text { Thickness at the pole dome, } s, \mathrm{~mm}\end{array}$ \\
\hline 0,5 & 1500 & $\approx 0,36$ \\
\hline 0,7 & 685 & $\approx 0,36$ \\
\hline
\end{tabular}




\section{2. Этап 2 - Подбор величины показателя $m^{*}$}

Известно из работы [6], что с увеличением величины показателя $m$ толщина в полюсе купола растет, и разнотолщинность уменьшается. По этой причине проводим расчеты показателя $m^{*}$ в среде программного комплекса ANSYS для того, чтобы добиться совпадения расчетного значения толщины в полюсе купола с экспериментально полученным значением.

При моделировании в ANSYS постановку и решение краевой задачи механики деформируемого твердого тела выполняем, используя теорию ползучести $[10,11]$.

В табл. 2 представлены значения толщины в полюсе купола высотой $H=35$ мм ( $\left.s_{\text {ANS }}\right)$, полученные в ANSYS при давлении $p=0,7 \mathrm{MПа,} \mathrm{и} \mathrm{значения} \mathrm{относительной} \mathrm{тол-}$ щины в полюсе купола $\left(s_{p}^{\prime}=s_{\text {ANS }} / s_{0}\right)$ для нескольких значений $m$ из интервала, содержащего значение $m_{0}=0,43$.

Используя данные табл. 2, при линейной интерполяции получаем $m^{*}=0,53$, а при квадратичной интерполяции $m^{*}=0,535$, для которых вычисляем новые значения К по формуле (6).

С найденными аналитическим путем постоянными материала, выполняем моделирование в ANSYS процесса СПФ полусферы до высоты $H=35$ мм и получаем значения толщины в полюсе купола (табл. 3).

Для дальнейших расчетов принимаем $m^{*}=0,53$.

\section{3. Этап 3 - Уточнение величины постоянной $K^{*}$}

Для расчета зависимости высоты купола от времени используем соотношение:

$$
t\left[\frac{p\left(R_{0}+r_{0}\right)}{2 K s_{0}}\right]^{1 / m}=2 I_{m}^{\prime}(\alpha) .
$$

Скорректированное значение постоянной $K^{\star}$ вычисляем по формуле:

$$
K^{*}=K\left(\frac{t_{\exp }}{t_{A N S}}\right)^{m^{*}} .
$$

На рис. 2 представлены зависимости относительной высоты купола $H^{\prime}$, где $H^{\prime}=H /\left(R_{0}+r_{0}\right)$ от времени формовки при постоянном давлении газа $\left(p_{1}=0,5 \mathrm{MПа}\right.$ и $p_{2}=0,7 \mathrm{MПа),} \mathrm{построенные} \mathrm{по} \mathrm{результатам} \mathrm{анали-}$ тических расчетов и полученные при моделировании

Табл. 2. Результаты расчета в ANSYS.

Table 2. The results of the calculation in ANSYS.

\begin{tabular}{|c|c|c|c|c|c|}
\hline$m$ & 0,4 & 0,429 & 0,45 & 0,5 & 0,6 \\
\hline$s_{-A N S}, \mathrm{~mm}$ & 0,318 & 0,330 & 0,336 & 0,353 & 0,376 \\
\hline$s_{p}^{\prime}, \mathrm{mm}$ & 0,318 & 0,330 & 0,336 & 0,353 & 0,376 \\
\hline
\end{tabular}

Табл. 3. Результаты расчета в ANSYS толщины в полюсе купола. Table 3. The results of the calculation in ANSYS thickness at the pole dome.

\begin{tabular}{|c|c|c|}
\hline & $m^{*}=0,53$ & $m^{*}=0,535$ \\
\hline$s_{\text {_ANS }}, \mathrm{mm}$ & 0,9754 & 0,9749 \\
\hline
\end{tabular}

в ANSYS при $m^{*}=0,53$ и $K^{*}=810.9 \mathrm{MПа} \cdot \mathrm{c}^{m}$. В эксперименте $H^{\prime}=0,972$. Видно, что численное решение при $p=0,7$ МПа проходит через экспериментальную точку.

На рис. 3 представлены зависимости относительной толщины в полюсе купола $s^{\prime}=s / s_{0}$ от его относительной высоты $H^{\prime}$ (a) и времени $t$, c (b), также построенные по результатам аналитических расчетов и полученные при моделировании в ANSYS.

Из рис. Зb также видно, что численное решение при $p=0,7$ МПа проходит через экспериментальную точку. Полученное согласие расчетных и экспериментальных данных связанно с тем, что на этапе 2 для подбо-

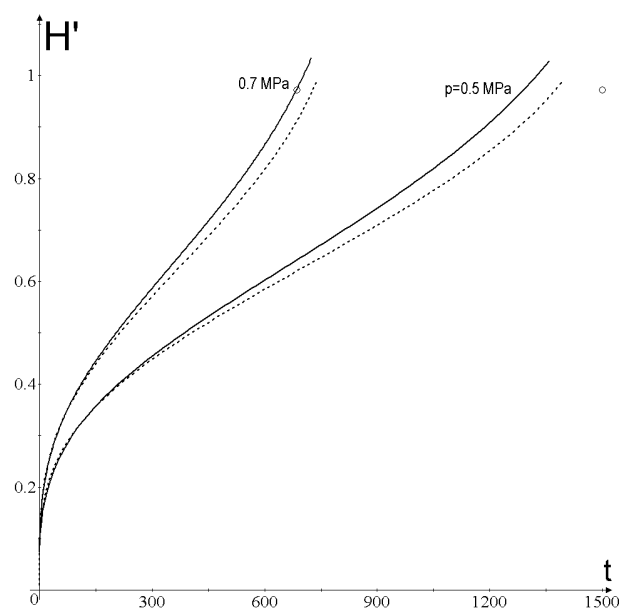

Pис. 2. Зависимости относительной высоты купола $H^{\prime}$ от времени $t$, с, вычисленные в ANSYS (сплошные линии) аналитически (пунктир) при различных значениях давления газа $p$, МПа (указано у кривых). Маркеры соответствуют экспериментальным значениям.

Fig. 2. Based on the relative height of the dome $H^{\prime}$ from the time $t, \mathrm{~s}$ computed in ANSYS (solid lines) and by the approximate formulas (dotted line) at different values of gas pressure $p, \mathrm{MPa}$ (indicated on curves). Markers correspond to experimental values.

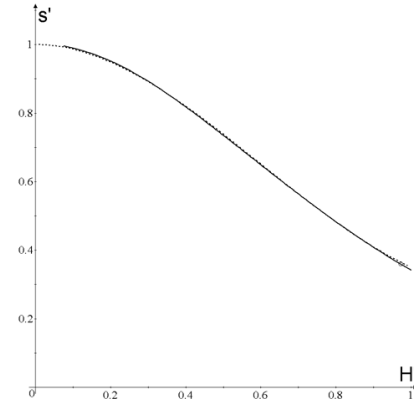

a

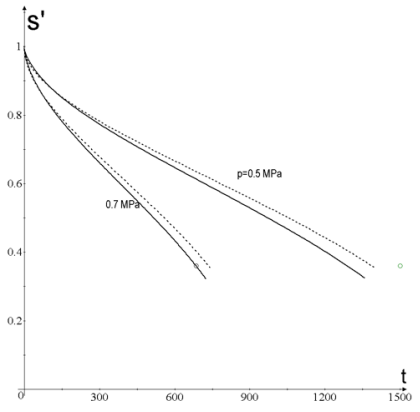

b
Рис. 3. Зависимости относительной толщины в полюсе купола $s^{\prime}$ от относительной высоты купола $H^{\prime}$ (a) и времени $t$, c (b), вычисленные в ANSYS (сплошные линии) и аналитически (пунктир) при различных значениях давления газа $p$, МПа (указано у кривых). Маркеры соответствуют экспериментальным значениям.

Fig. 3. Based on the relative thicknesses at the pole dome $s^{\prime}$ from the relative height of the dome $H^{\prime}$ (a) and time $t$, s (b) calculated in ANSYS (solid lines) and by the approximate formulas (dotted line) at different values of gas pressure $p, \mathrm{MPa}$ (indicated on curves). Markers correspond to experimental values. 
ра значения $m^{*}$ моделирование в ANSYS процесса СПФ проводили при значении давления $p=0,7$ МПа. При значении давления $p=0,5$ МПа погрешность вычислений составляет $8,6 \%$.

\section{3. Выводы}

Предлагаемая в настоящей работе методика определения постоянных материала $K$ и $m$, входящих в стандартную модель СП $\sigma=K \xi^{m}$, позволяет получить приемлемый для инженерных расчетов результат, используя набор экспериментальных данных двух тестовых формовок: $\left\{t_{i}, p_{i}, h_{i}, s_{i}\right\}, i=1,2$, где $t_{i}$ - продолжительность формовки полусферы при давлении газа $p_{i}=$ const до высоты купола $h_{i}$ и толщина в полюсе купола $s_{i}$.

\section{Литература/References}

1. W. A. Backofen, I. R. Turner, D. H. Avery. Superplasticity in an Al Zn Alloy. Trans. ASM.1964. V. 57. pp.980-990.

2. Kaibyshev O.A. Superplasticity of industrial alloys. Metallurgy. 1984. p. 264. (in Russian) [O.А. Кайбышев. Сверхпластичность промышленных сплавов. Металлургия. 1984. с. 264.]

3. F.U. Enikeev and A.A. Kruglov. An analysis of the superplastic forming of a thin circular diaphragm. International Journal of Mechanical Sciences. 1995. Vol.37. No.5. pp.473-483.

4. R.A. Vasin, F. U. Enikeev, A.A. Kruglov, R. V. Safiullin. Identification of defining relations according to the results of technological experiments. Izv. RAN, Mechanics of solids. 2003. No. 2. pp. 111-123. (in Russian) [Р.А. Васин, Ф.У. Еникеев, А.А. Круглов, P.В. Сафиуллин. Об идентификации определяющих соотношений по результатам технологических экспериментов. Изв. РАН, Механика твердого тела. 2003. № 2. c. $111-123$.

5. S.A. Aksenov, E.N. Chumachenko, A.V. Kolesnikov, S.A. Osipov Determination of optimal gas forming conditions from free bulging tests at constant pressure.
Journal of Materials Processing Technology. 2015. pp.158- 164 .

6. F.S. Jarrar, F.K. Abu-Farha, Jr.L. G. Hector, M.K. Khraisheh. Simulation of High-Temperature AA5083 Bulge Forming with a Hardening. Softening Material Model. Journal of Materials Engineering and Performance. DOI: $10.1007 /$ s11665-008-9322-5

7. L. Carrino, G. Giuliano, C. Palmieri. On the optimization forming processes by the finite- elerment method. Jurnal of Meterials Processing Technology. 2003. pp. 373 - 377.

8. F. U. Enikeev, O. P., Tulupov, V. R. Ganiev, A. K. Shmakov, A.V. Kolesnikov. Determination of the superplastic properties of aluminum alloys according to the results of the test molds round of membranes at constant pressure. KSHP. OMD. 2015. No.11. pp. 7-11. (in Russian) [Ф.У. Еникеев, О.П. Тулупова, В. Р. Ганиева, А.К. Шмаков, А.В. Колесников. Определение сверхпластических свойств алюминиевых сплавов по результатам тестовых формовок круглых мембран при постоянном давлении. КШП. ОМД. 2015. № 11. с. $7-11]$

9. G.G. Cornfield and R.H. Johnson. The Forming of Superplastic Sheet Materials. Int. J. Mech. Sci. 1970. V.12, № 6. P.479-490.

10. O.P. Tulupova, A.A. Slesareva, A.A. Kruglov, F. U. Enikeev. Peculiarities of deforming a rectangular edge welded envelope made of commercial titanium alloy. Letters on materials. 2015.5 (4). pp. $478-481$.

11. A.Y. Samoylova, V.R. Ganieva, F.U. Enikeev, A.A. Kruglov. Analysis of the stress-strain state in the deformation zone during superplastic forming of circular membrane Part II. Modeling of the process of superplastic forming. Letters on materials. 2013. V. 3 (3). pp.252-256. (in Russian) [А.Ю. Самойлова, В. Р. Ганиева, Ф. У. Еникеев, А. А. Круглов. Анализ напряженно-деформированного состояния в очаге деформации при сверхпластической формовке круглой мембраны Часть II. Моделирование процесса сверхпластической формовки. Письма о материалах. 2013. т. 3 (3). pp. $252-256]$. 